\title{
Strong motion PGA prediction for southwestern China from small earthquake records
}

\author{
Zhengru Tao ${ }^{1}$, Xiaxin Tao $^{2,1}$, and Anping Cui ${ }^{1}$ \\ ${ }^{1}$ Key Laboratory of Earthquake Engineering and Engineering Vibration, Institute of Engineering Mechanics, \\ China Earthquake Administration, Harbin, China \\ ${ }^{2}$ Harbin Institute of Technology, Harbin, China \\ Correspondence to: Zhengru Tao (taozhengru@aliyun.com)
}

Received: 9 July 2015 - Published in Nat. Hazards Earth Syst. Sci. Discuss.: 4 September 2015

Revised: 5 April 2016 - Accepted: 10 April 2016 - Published: 20 May 2016

\begin{abstract}
For regions without enough strong ground motion records, a seismology-based method is adopted to predict motion PGA (peak ground acceleration) values on rock sites with parameters from small earthquake data, recorded by regional broadband digital monitoring networks. Sichuan and Yunnan regions in southwestern China are selected for this case study. Five regional parameters of source spectrum and attenuation are acquired from a joint inversion by the micro-genetic algorithm. PGAs are predicted for earthquakes with moment magnitude $\left(M_{\mathrm{w}}\right)$ 5.0, 6.0, and 7.0 respectively and a series of distances. The result is compared with limited regional strong motion data in the corresponding interval $M_{\mathrm{w}} \pm 0.5$. Most of the results ideally pass through the data clusters, except the case of $M_{\mathrm{w}} 7.0$ in the Sichuan region, which shows an obvious slow attenuation due to a lack of observed data from larger earthquakes $\left(M_{\mathrm{w}} \geq 7.0\right)$. For further application, the parameters are adopted in strong motion synthesis at two near-fault stations during the great Wenchuan Earthquake M8.0 in 2008.
\end{abstract}

\section{Introduction}

Ground motion prediction equation (GMPE) is a vital field in the research of engineering seismology. A great number of research results have been published, and most of them are empirical, such as those for the western United States by the Next Generation Attenuation (NGA) project (Power et al., 2008; Bozorgnia et al., 2012; Boore et al., 2014) and those for Japan (Si and Midorikawa, 2000; Kanno, 2006). Empirical GMPEs are developed, mainly based on plenty of strong ground motion records. For the regions without enough observed data, like the mainland of China and many others, it is difficult to build GMPEs this way. Obviously, there are more records of small earthquakes from regional earthquake monitoring networks than those of strong motion in general. The main goal of this paper is to see if these data could be a basis to estimate the regional parameters in a seismology-based attenuation relationship (Hanks and McGuire, 1981; Boore, 1983; Atkinson, 1984; Boore and Atkinson, 1987). If they are really helpful, the bottleneck restriction of insufficient strong motion records will be broken completely. Five regional parameters, stress drop $\Delta \sigma, Q_{0}$ and $\eta$ in a quality factor, and $R_{1}$ and $R_{2}$ in a geometric attenuation factor, are acquired from a joint inversion by the micro-genetic algorithm. The objective function is defined as the summation of square differences between envelopes of calculated and observed Fourier velocity amplitude spectra. The result is compared with the limited observed PGA (peak ground acceleration) data that have never been used in the inversion, to show the feasibility of this method. The regional parameters are further adopted in synthesis of strong motions at two near-fault rock stations with a hybrid source model for magnitude 8.0. The result is compared with the records during the great Wenchuan Earthquake in 2008.

\section{Database}

\subsection{Data for inversion of the five parameters}

Small earthquake data recorded from January 2001 to December 2007 by Sichuan and Yunnan digital earthquake net- 


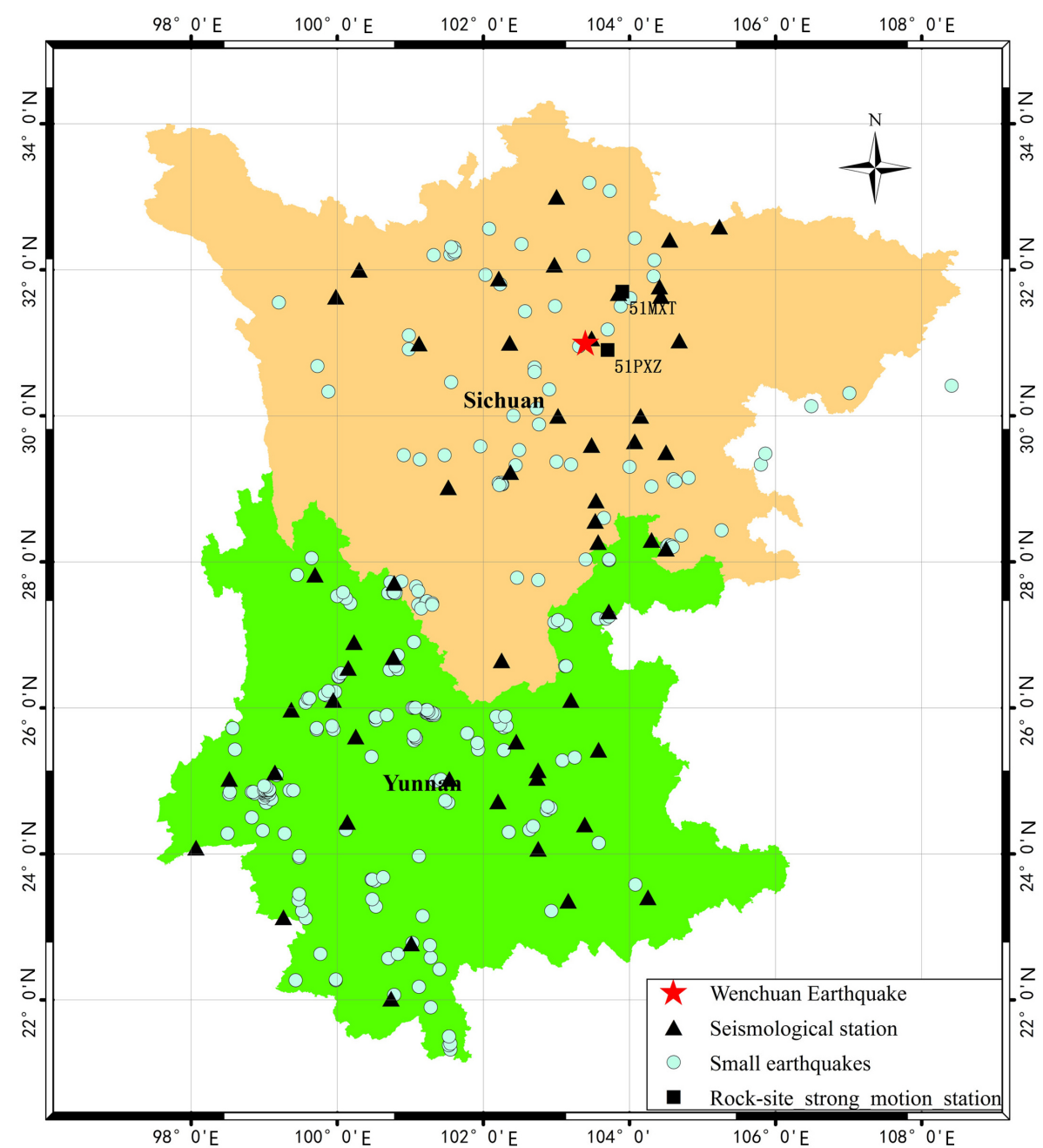

Figure 1. Seismological stations and small earthquakes in Sichuan and Yunnan regions.

works are adopted for the inversion. The period is from the beginning of operations of both networks to the end of the year before the Wenchuan Earthquake. There are 29 digital seismological stations in Sichuan and 26 stations in Yunnan; their locations are shown as triangles in Fig. 1.

A total of 147 records from 82 small earthquakes in Sichuan and 863 records from 154 small earthquakes in Yunnan, with $M_{\mathrm{w}} 3.5 \sim 4.5$ and focal depth $\leq 30 \mathrm{~km}$, are downloaded from the China Earthquake Networks Center (2011) and processed using a conventional procedure. The epicenters are shown as circles in Fig. 1.

The distributions of focal depth and hypocentral distance with $M_{\mathrm{w}}$ are shown in Figs. 2 and 3, respectively. The hypocentral distances distribute more or less uniformly from 50 to $300 \mathrm{~km}$ in the regions.

The time window of the velocity time history, with time interval $0.02 \mathrm{~s}$, starts from S-wave arrives until that $80 \%$ energy is included.

\subsection{Limited strong motion data for checkout}

There are 69 strong ground motion stations in Sichuan and 36 stations in Yunnan. A total of 1234 records from 118 Sichuan earthquakes and 78 records from 27 Yunnan earthquakes, with $M_{\mathrm{w}} \geq 4.5$ and focal depth $\leq 30 \mathrm{~km}$, are adopted. The processed records are directly downloaded from the China Strong Motion Networks Center. The strong ground motion data of Sichuan are all from aftershocks of the Wenchuan Earthquake. The distributions of $M_{\mathrm{w}}$ focal depth and $M_{\mathrm{w}}$ hypocentral distance are shown in Figs. 4 and 5. The focal depth is from 8 to $20 \mathrm{~km}$ in Sichuan, and most focal depths of big earthquakes in Yunnan are less than $15 \mathrm{~km}$. Hypocentral distances in Sichuan are from 50 to $300 \mathrm{~km}$, and most distances in Yunnan are less than $120 \mathrm{~km}$; some of them are less than $20 \mathrm{~km}$. 

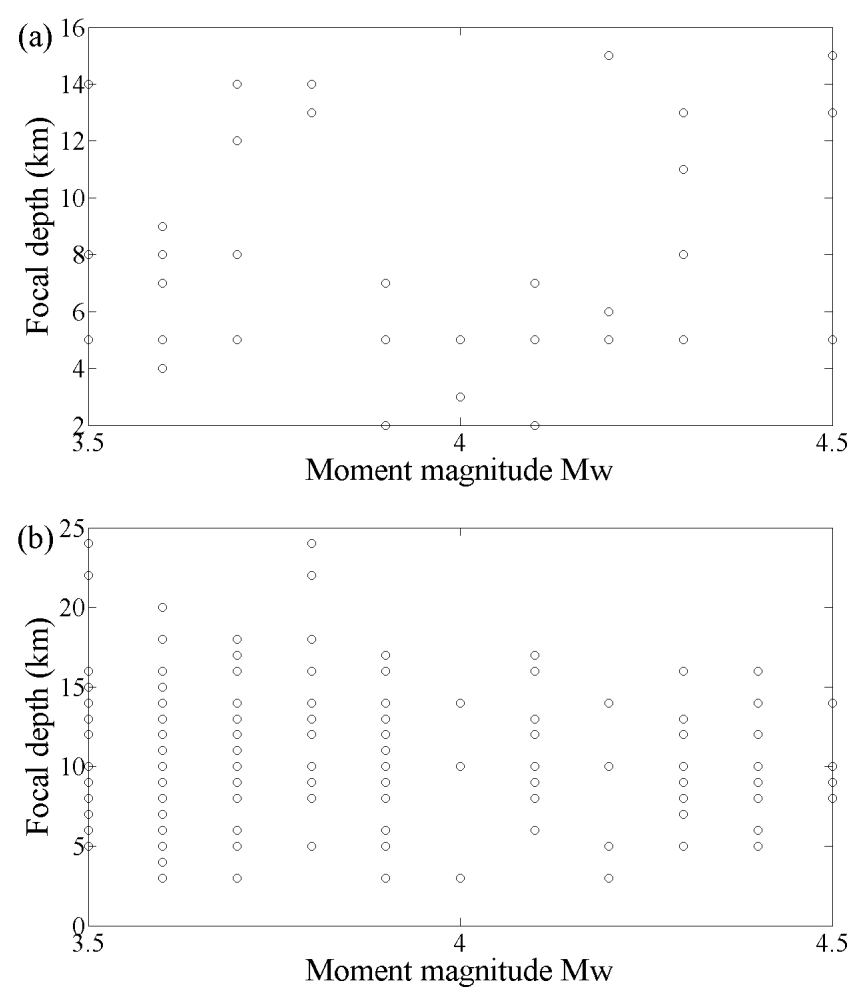

Figure 2. $M_{\mathrm{W}}$ focal depth distribution of small earthquake records in (a) Sichuan region and (b) Yunnan region.

\section{Methodology}

Fourier amplitude spectrum of ground motion from a point source of a given earthquake can be presented as (Boore, 1983; Atkinson and Boore, 2000; Boore, 2003)

$\mathrm{FA}\left(M_{0}, f, R\right)=C \cdot S\left(M_{0}, f\right) \cdot G(R) \cdot D(R, f)$

$$
\cdot A(f) \cdot P(f) \cdot I(f)
$$

where $C$ is a constant, as Eq. (2); $M_{0}$ is the corresponding seismic moment; $f$ is the frequency; $R$ is the distance; $S(\cdot)$ is the source spectrum; $G(\cdot)$ is the geometric attenuation term; $D(\cdot)$ is the medium energy dissipation term; $A(\cdot)$ is the near-surface transfer function with $\bar{V}_{30} 620 \mathrm{~m} \mathrm{~s}^{-1}$ for generic rock (Boore and Joyner, 1997); $P(\cdot)$ is the high-frequency filter with $f_{\max }=5 \mathrm{~Hz}$ from regional strong ground motion records; $I(\cdot)$ is the motion type conversion function for displacement, velocity and acceleration.

The constant, proportion factor, is given by

$C=\frac{R_{\theta \varphi} F V}{4 \pi R_{0} \rho_{\mathrm{s}} \beta_{\mathrm{s}}^{3}}$,

where $R_{\theta \phi}$ is the radiation pattern of the shear excitation, with the assumption that the energy was equally partitioned into two horizontal components, $R_{\theta \phi}=0.6 ; F$ is the free surface effect, $F=2.0 ; V$ is the vectorial partitioning of shear-wave energy into two components of equal amplitude,
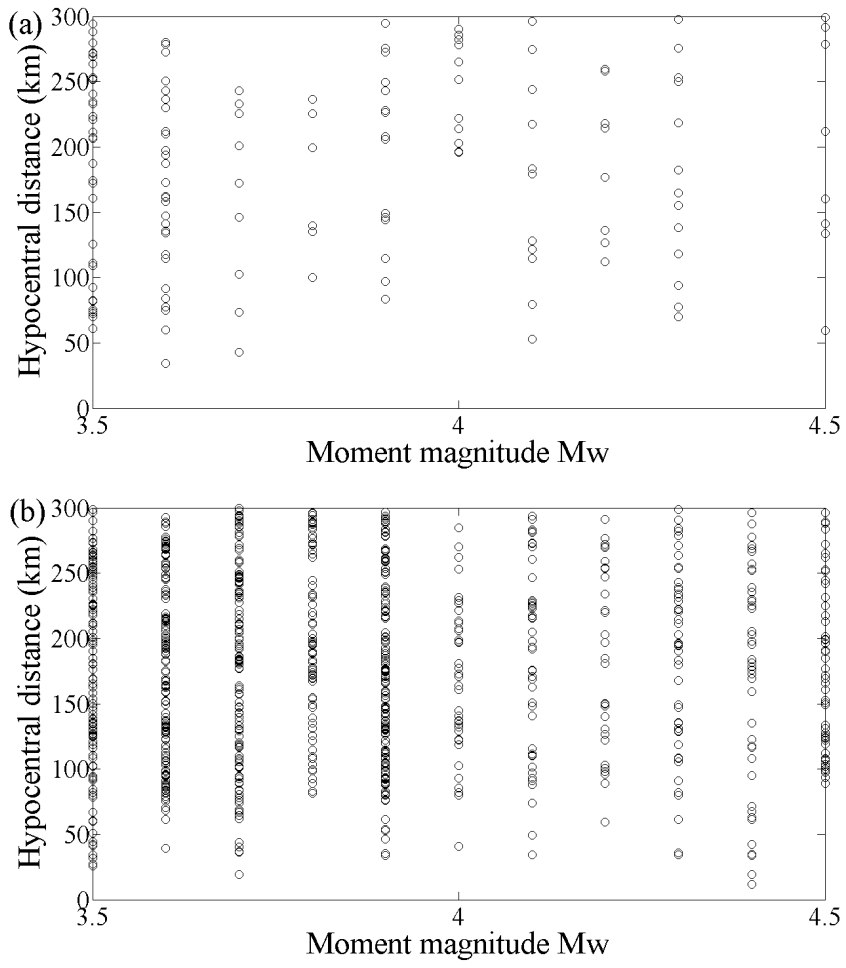

Figure 3. $M_{\mathrm{W}}$ hypocentral distance distribution of small earthquake records in (a) Sichuan region and (b) Yunnan region.

$V=\frac{1}{\sqrt{2}}$ (McGuire and Hanks, 1980); $R_{0}$ is a reference distance, $R_{0}=1 \mathrm{~km}$ (Boore, 2003); $\rho_{\mathrm{S}}$ is the density in the vicinity of the source, $\rho_{\mathrm{s}}=2.8 \mathrm{~g} \mathrm{~cm}^{-3} ; \beta_{\mathrm{s}}$ is the shear-wave velocity in the vicinity of the source, $\beta_{\mathrm{s}}=3.5 \mathrm{~km} \mathrm{~s}^{-1}$ (Wu et al., 2001; Xie et al., 2012).

In order to eliminate the dependence of the motion amplitude on source size, the following source spectrum model (Tao et al., 2008) is adopted rather than the well-known $\omega^{2}$ spectrum (Brune, 1970) or the two corner frequency spectrum (Atkinson, 1993).

$S\left(M_{0}, f\right)=\frac{M_{0}}{\left[1+\left(\frac{f}{f_{c}}\right)^{a}\right]^{b}}$,

where $f_{\mathrm{c}}$ is the corner frequency, estimated by Eq. (4) (Boore, 2003); parameter $a$ and $b$ could be taken as $a=$ $3.05-0.33 M, b=2.0 / a$.

$f_{\mathrm{c}}=4.9 \times 10^{6} \times \beta_{\mathrm{s}} \times\left(\Delta \sigma / M_{0}\right)^{1 / 3}$,

where $\Delta \sigma$ is the dynamic stress drop.

The geometric attenuation term $G(R)$ is commonly adopted as in Eq. (5), for the distance ranges predominated by the shear wave, the surface wave, and by the wave guide effect in between these two (Atkinson and Mereu, 1992; 
Table 1. Inverse results.

\begin{tabular}{llllll}
\hline Parameters & $\Delta \sigma$ (bars) & $Q_{0}$ & $\eta$ & $R_{1}(\mathrm{~km})$ & $R_{2}(\mathrm{~km})$ \\
Range for searching & $40-200$ & $90-400$ & $0.2-0.8$ & $50-100$ & $100-150$ \\
\hline Sichuan & 85 & 155 & 0.6804 & 87 & 120 \\
Yunnan & 72 & 164 & 0.6647 & 83 & 122 \\
\hline
\end{tabular}
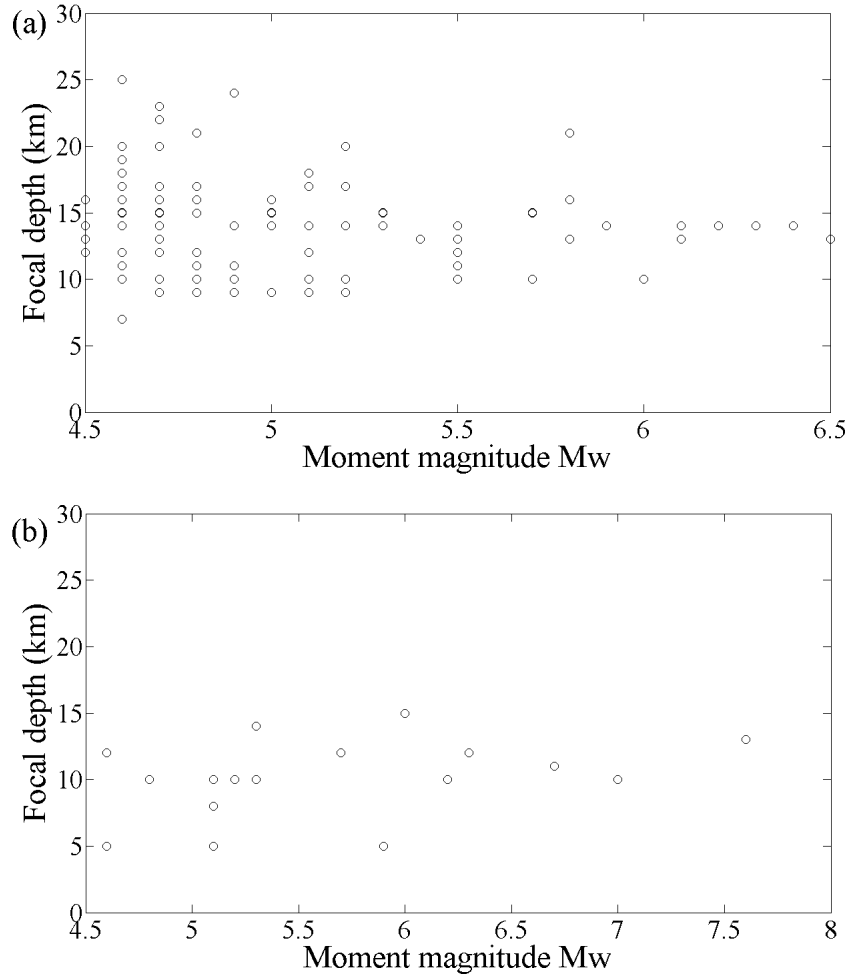

Figure 4. $M_{\mathrm{W}}$ focal depth distribution of strong ground motion records in (a) the Sichuan region and (b) the Yunnan region.

Atkinson and Boore, 1995).

$G(R)= \begin{cases}\frac{1}{R} & R \leq R_{1} \\ \frac{1}{R_{1}} & R_{1} \leq R \leq R_{2} \\ \frac{1}{R_{1}} \sqrt{\frac{R_{2}}{R}} & R_{2} \leq R,\end{cases}$

where $R_{1}$ and $R_{2}$ are regional geometric attenuation parameters, and are concerned with the regional structure of the earth's crust and dynamic characteristics of the crust medium.

The energy dissipation along the wave propagation path is very complicated; the medium energy dissipation term $D(R, f)$ can be taken as (Boore, 1983)

$D(R, f)=e^{-\frac{\pi \cdot f \cdot R}{Q(f) \cdot \beta}}$
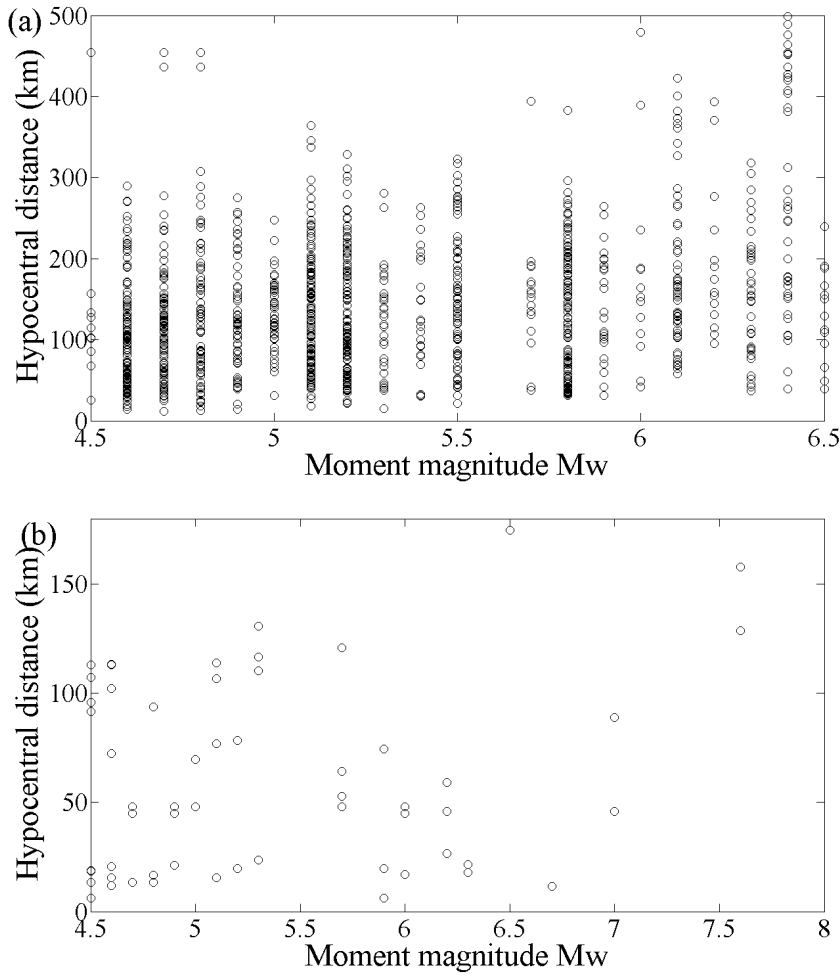

Figure 5. $M_{\mathrm{W}}$ hypocentral distance distribution of strong ground motion records in (a) the Sichuan region and (b) the Yunnan region.

where $Q(f)$ is the quality factor, and can be described as (Beresnev, 1998)

$Q=Q_{0} f^{\eta}$,

where $Q_{0}$ and $\eta$ are the region-related coefficient and the exponent, respectively.

The above-mentioned five regional parameters, $\Delta \sigma, R_{1}$, $R_{2}, Q_{0}$, and $\eta$, could not be measured directly. In general, $\Delta \sigma, Q_{0}$, and $\eta$ obtain values from inversion of regional far field records; $R_{1}$ and $R_{2}$ are estimated from the regional earth crust structure. In practice, there are several different values for the former three parameters from different authors with different data, even different forms of the formula in some regions, but no values are published in other regions. It is also not easy to estimate the values of the latter two in some regions with complex topography, such as in Sichuan and Yunnan. Therefore, the authors of this paper suggested a joint inversion procedure by the micro-genetic algorithm, $\mu \mathrm{GA}$ 

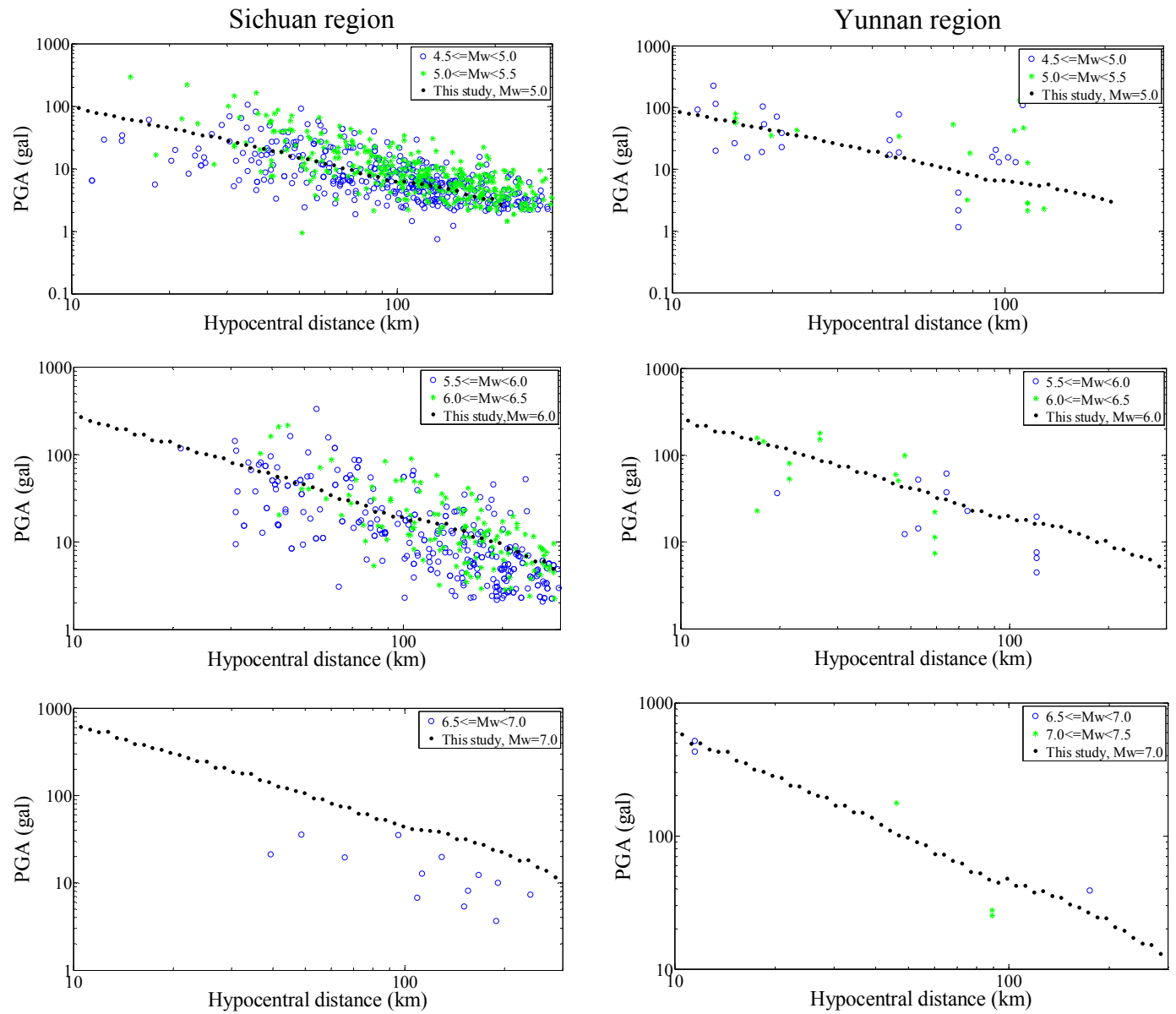

Figure 6. Comparison of the result with strong ground motion data in the Sichuan region and the Yunnan region.

(Carroll, 2009), to see if the records of small earthquakes are helpful. In nature, it is a search scheme. The five values are searched from the pre-chosen ranges that are large enough to cover all possible values (Atkinson and Boore, 1995; Qiao et al., 2006; Mao et al., 2005; Zhang et al., 2007; Qin and Kan, 1986; Su et al., 2006; Liu, 2005; Hu et al., 2003), by minimizing the objective function defined in Eq. (8).

$\varphi_{j}=\sum_{i=1}^{4096} \sum_{k=1}^{N}\left[\mathrm{FA}_{0}(i, k)-\mathrm{FA}_{j}(i, k)\right]^{2}$,

where $\mathrm{FA}_{0}$ is the $i$ th amplitude of enveloped Fourier amplitude spectrum of the $k$ th record; $\mathrm{FA}_{j}(i, k)$ is the corresponding amplitude by Eq. (1) and the $j$ th generation parameter values; 4096 is the total amplitude number of the Fourier spectrum from the time step $0.02 \mathrm{~s}$ and the corresponding frequency spacing $0.0122 \mathrm{~Hz} ; N$ is the total number of the records.

The five parameter values of the solution, after having searched 2000 generations, are listed in Table 1.

For each given pair of magnitude and distance, the Fourier amplitude spectrum by Eq. (1) with the parameter values in Table 1, combined with a random phase spectrum, is transformed into a time domain. The time history is then enveloped for the amplitude nonstationarity. Then, the enveloped time history is transformed back into frequency domain, and scaled to the amplitudes directly from Eq. (1). The scaled spectrum is transformed back to the time domain again, and PGA for this magnitude distance is read from this final time history. Fifty acceleration time histories are generated and the average PGA is the stable expected PGA.

\section{Results and discussion}

\subsection{Strong ground motion prediction in Sichuan and Yunnan regions}

It is a kind of check, to compare the result with regional limited strong ground motion data in Sect. 2.2. These data are never adopted in the inversion. The attenuation curves for $M_{\mathrm{w}} 5.0,6.0$, and 7.0, with data points in the intervals $M_{\mathrm{w}} \pm 0.5$, are shown in Fig. 6. In the cases of $M_{\mathrm{w}} 5.0$ and 6.0 , the result curves pass through the corresponding strong motion data, and the data points are distributed evenly on 

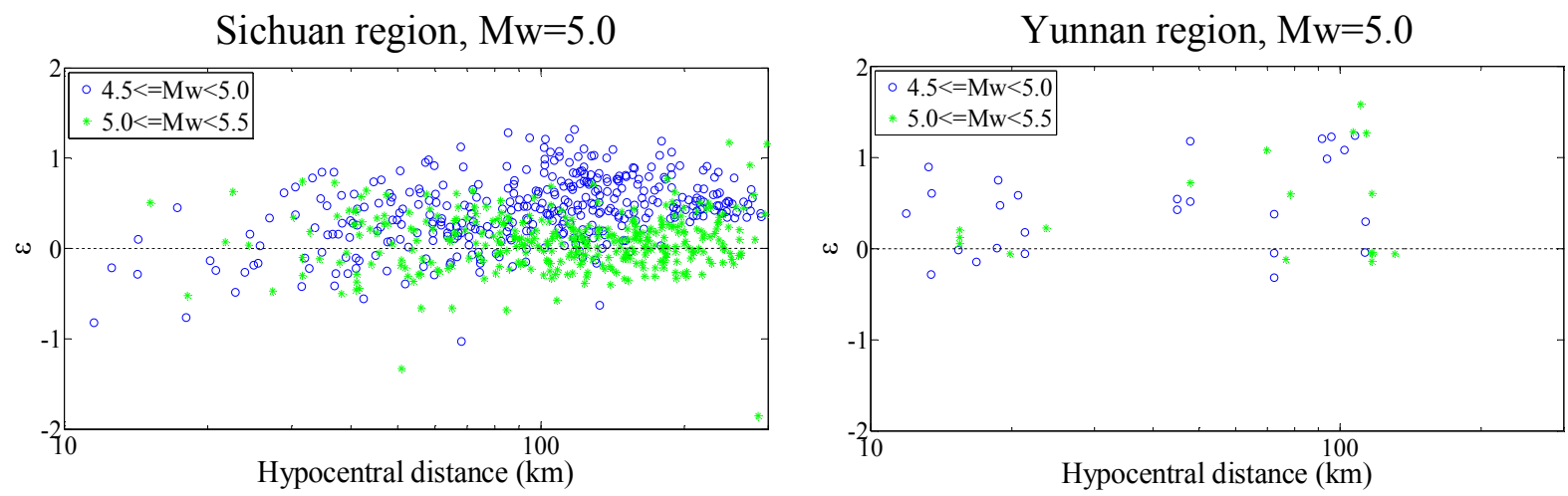

Sichuan region, $\mathrm{Mw}=6.0$

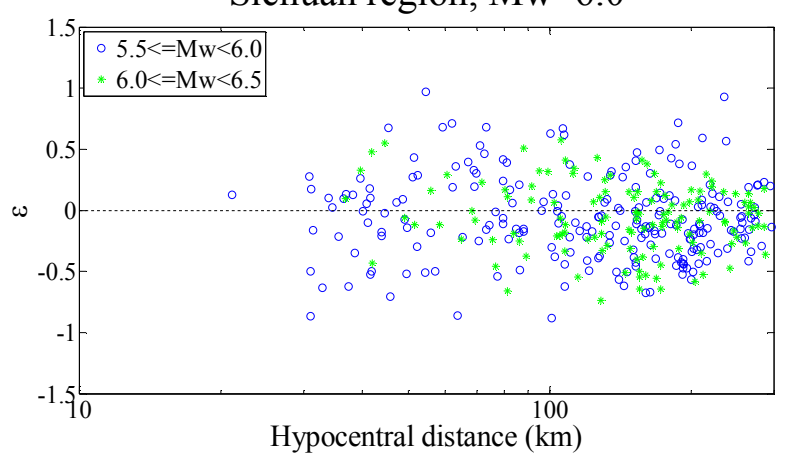

Yunnan region, $\mathrm{Mw}=6.0$

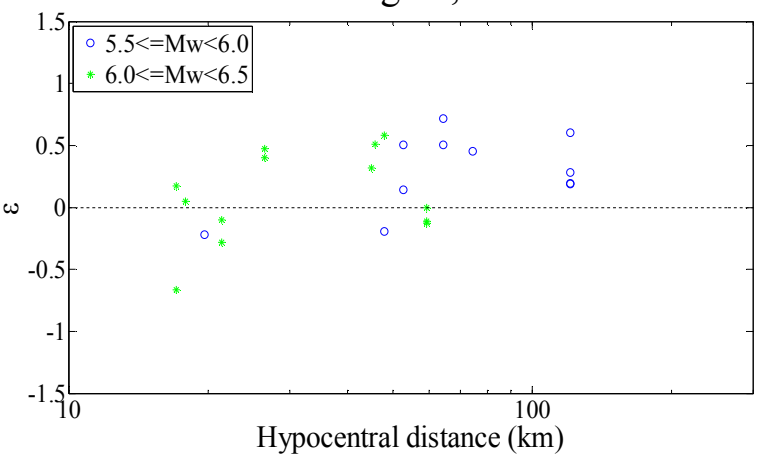

Sichuan region, $\mathrm{Mw}=7.0$

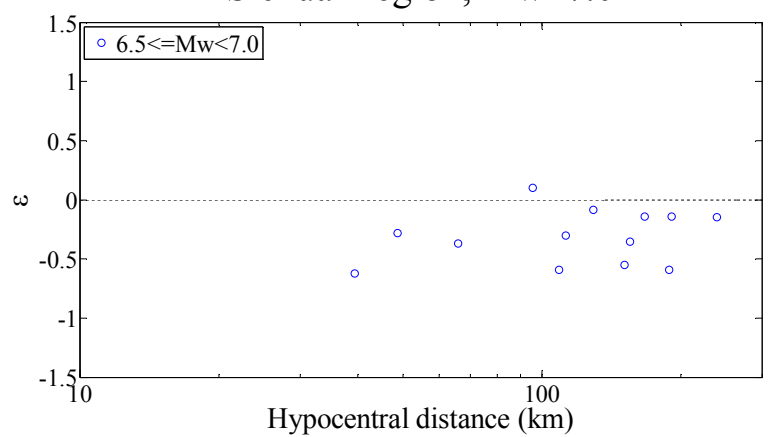

Yunnan region, $\mathrm{Mw}=7.0$

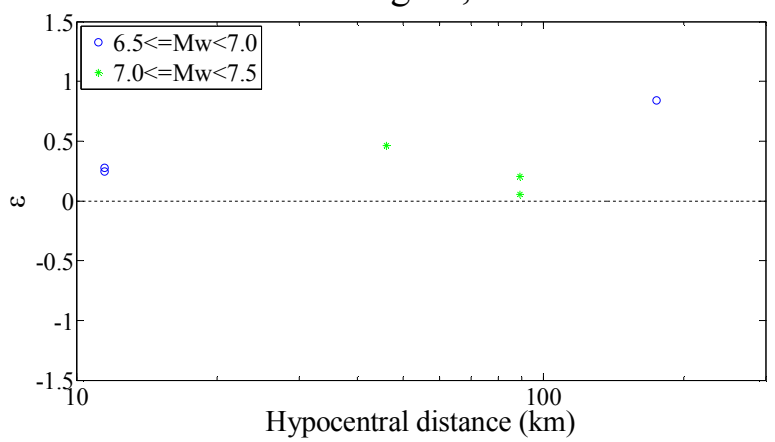

Sichuan region
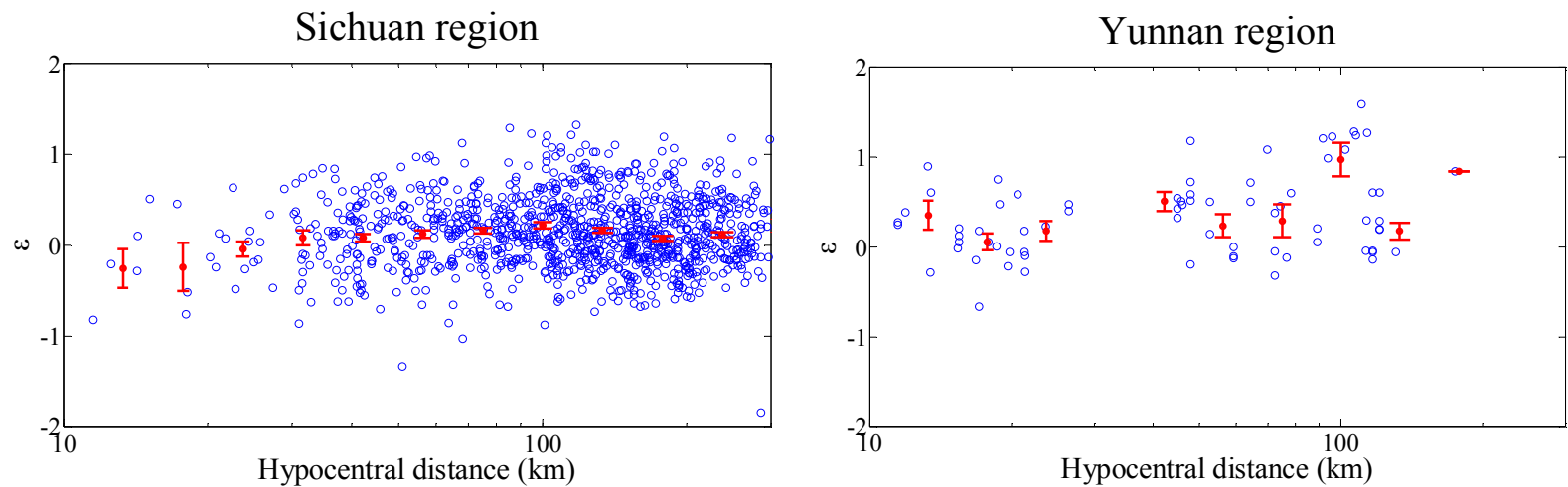

Figure 7. Residual distribution in the Sichuan region and the Yunnan region. 
(a)

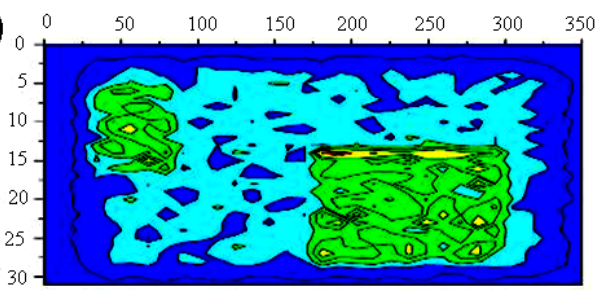

(c)

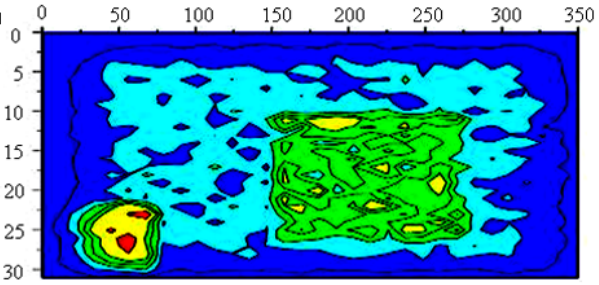

(e)

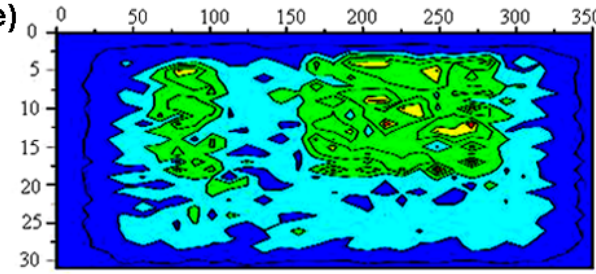

(g)

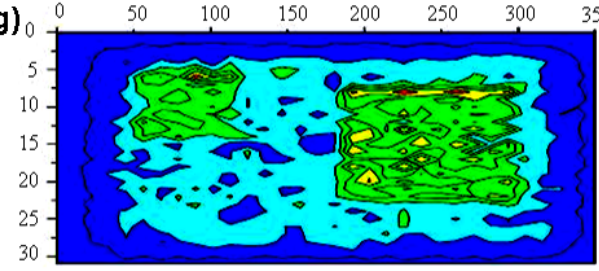

(i)

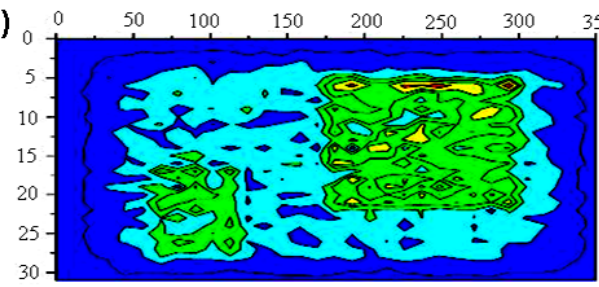

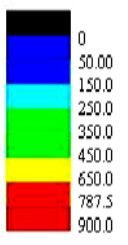
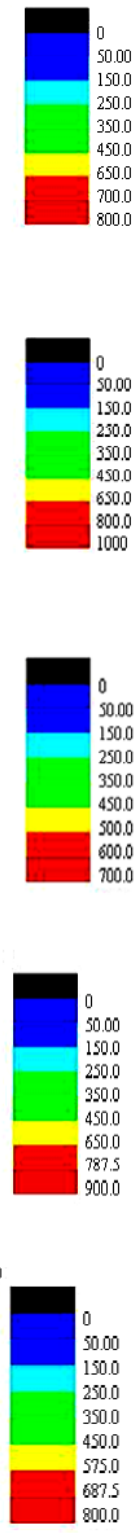

(b)

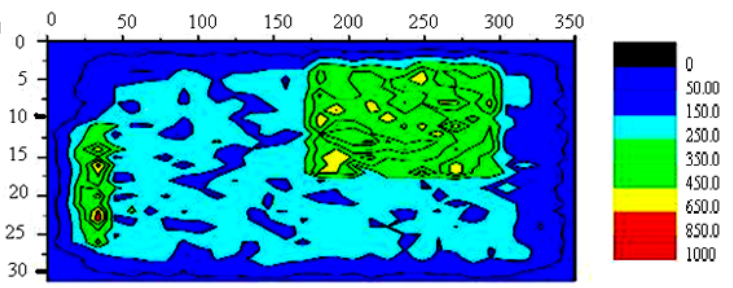

(d)

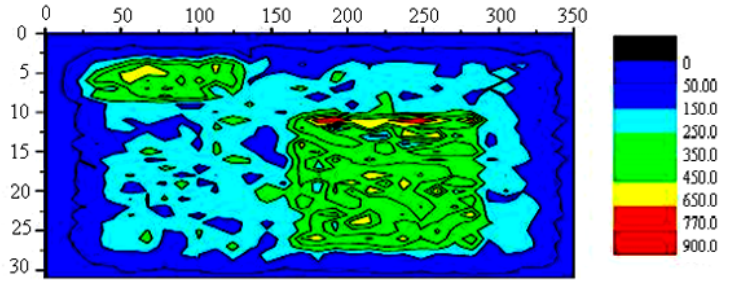

(f)

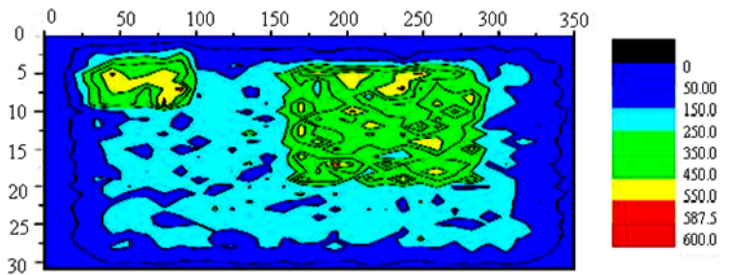

(h)

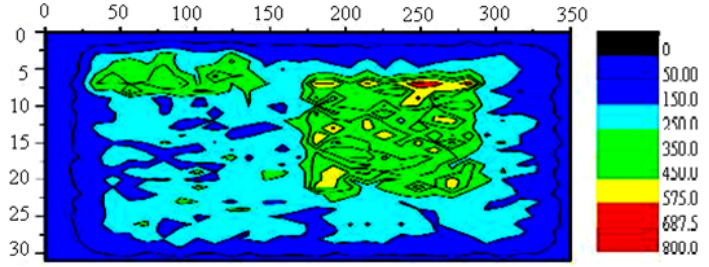

(j)

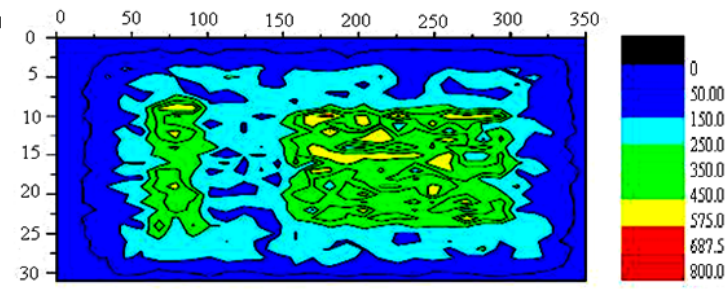

Figure 8. Ten hybrid source models in the case of Wenchuan Earthquake, redrawn from Liu and Tao (2013).

both sides of the curve, which means our results present the mean ground motion levels well. In the case of $M_{\mathrm{w}} 7.0$, our result for the Sichuan region is obviously higher than the observed data, since we do not have data showing magnitudes of more than $M_{\mathrm{w}} 6.5$ there.

To get a quantitative measure, the residual between the observed value and the corresponding predicted value is calculated by

$\varepsilon=\log _{10}\left(\frac{\mathrm{PGA}}{\mathrm{PGA}^{\prime}}\right)=\log _{10} \mathrm{PGA}-\log _{10} \mathrm{PGA}^{\prime}$,

where PGA is the observed value; PGA' is the corresponding predicted value. Distributions of $\varepsilon$ for the cases $M_{\mathrm{W}} 5.0,6.0$, and 7.0 are shown in Fig. 7. The two figures, at the bottom in Fig. 7, show the $\varepsilon$ distribution for all magnitude range, within bins equally spaced with respect to $\log _{10} R$.

In the cases of $M_{\mathrm{w}} 5.0$ and $6.0, \varepsilon$ values are evenly distributed around the mean of 0.0 which means the ratios of the observed values and the predicted values are around 1 . Most residuals of $M_{\mathrm{w}} 7.0$ in the Sichuan region are lower than 0.0 because of the same reason as mentioned above. No perceptible trend indicates that the path terms could represent the data trends reasonably, which is the same as the conclusion in Boore et al. (2014). 

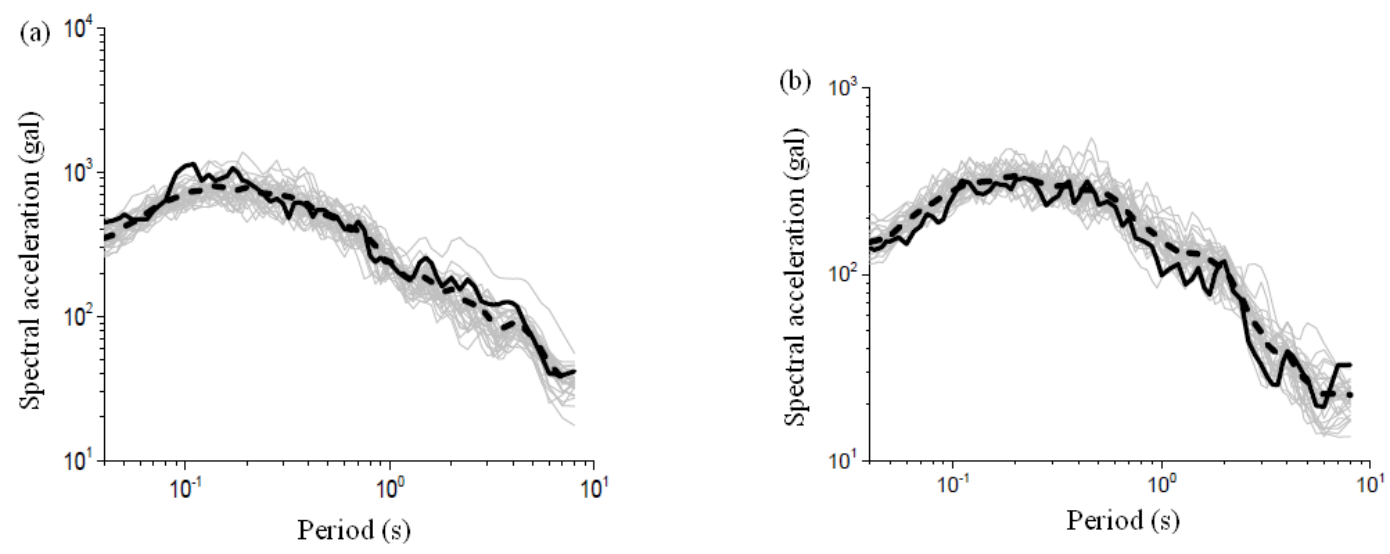

Figure 9. Acceleration response spectra of motions from 30 hybrid source models at (a) Maoxian station (51MXT) and (b) Pixian station (51PXZ), redrawn from Tao et al. (2013).
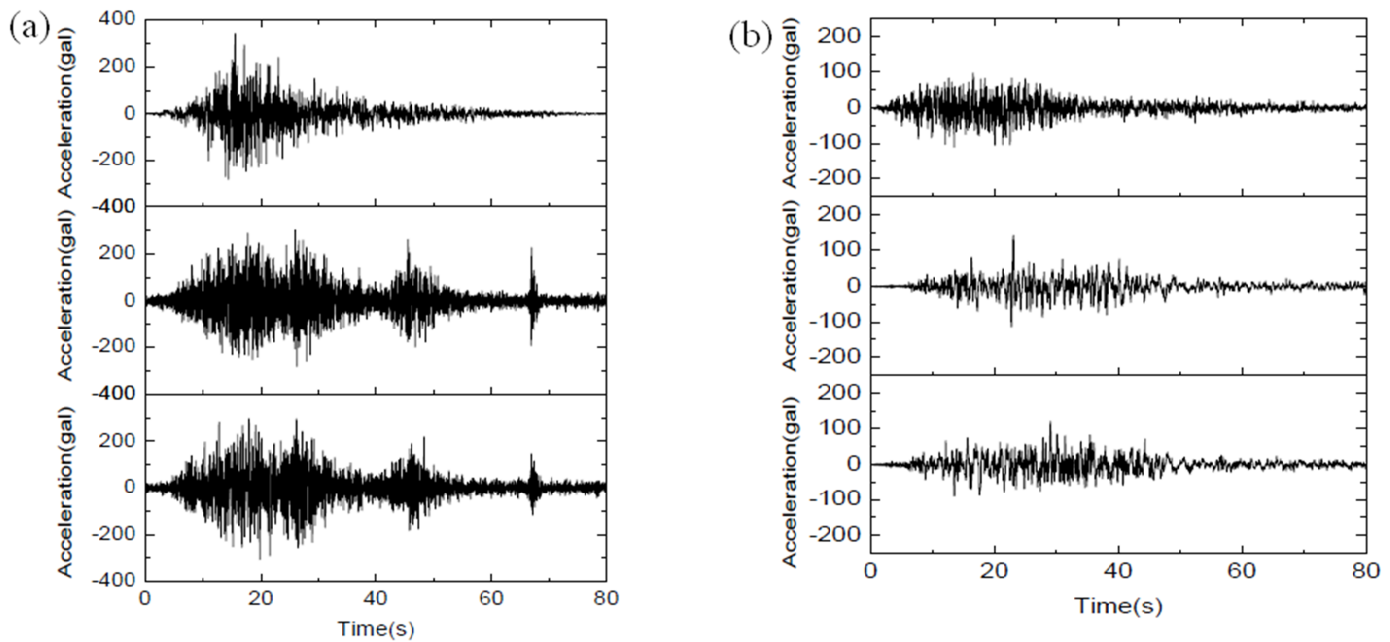

Figure 10. Acceleration time histories synthesized and observed at (a) Maoxian station (51MXT) and (b) Pixian station (51PXZ), redrawn from Tao et al. (2013).

The mean value and standard deviation for all distances but the three magnitude intervals are listed in Table 2. The statistic features are stable as a whole and unusual in just a few intervals. It means that there are really not enough observed data in the interval. The values of standard deviation are comparable with those of empirical prediction in the regions with rich strong motion data. The facts show that it is possible to build an attenuation relation by small earthquake data recorded by regional broadband earthquake networks, for regions without enough strong ground motion records.

\subsection{Application of the regional parameters to predict near-fault strong motion}

Near-fault ground motion of a large earthquake is complicated and governed by the source mechanism; therefore, it is not possible to be predicted by GMPEs, but possible by
Table 2. Mean value and standard deviation of residuals.

\begin{tabular}{l|ll|ll|ll}
\hline \multirow{2}{*}{} & \multicolumn{2}{|c|}{$M_{\mathrm{w}}=5.0$} & \multicolumn{2}{c|}{$M_{\mathrm{w}}=6.0$} & \multicolumn{2}{c}{$M_{\mathrm{w}}=7.0$} \\
\cline { 2 - 7 } Regions & $\begin{array}{l}\text { Mean } \\
\text { value }\end{array}$ & $\begin{array}{l}\text { Standard } \\
\text { deviation }\end{array}$ & $\begin{array}{l}\text { Mean } \\
\text { value }\end{array}$ & $\begin{array}{l}\text { Standard } \\
\text { deviation }\end{array}$ & $\begin{array}{l}\text { Mean } \\
\text { value }\end{array}$ & $\begin{array}{l}\text { Standard } \\
\text { deviation }\end{array}$ \\
\hline Sichuan & 0.08 & 0.30 & -0.08 & 0.33 & -0.53 & 0.22 \\
Yunnan & 0.21 & 0.56 & -0.09 & 0.31 & 0.05 & 0.16 \\
\hline
\end{tabular}

strong motion synthesis from a finite fault source model. In order to see if the five regional parameters obtained above are helpful in near-fault ground motion synthesis, two rocksite stations, Maoxian station (MXT, 31.7 $\left.\mathrm{N}, 103.9^{\circ} \mathrm{E}\right)$ and Pixian station (PXZ, $30.9^{\circ} \mathrm{N}, 103.7^{\circ} \mathrm{E}$ ), shown as squares in Fig. 1, in the areas with intensity IX and VIII, respectively, during the M8.0 Wenchuan Earthquake, are selected as examples, since strong ground motion was recorded there dur- 

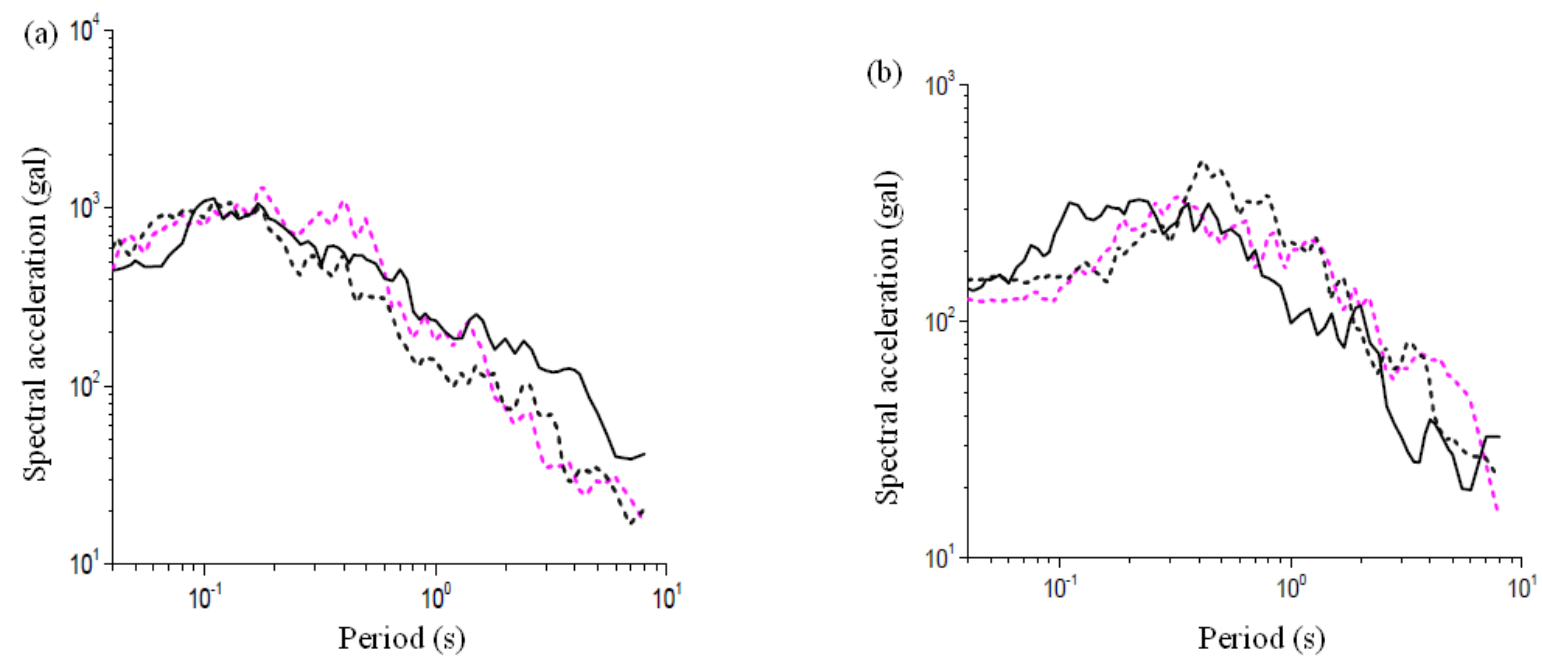

Figure 11. The mean acceleration response spectra of synthesized and observed motions at (a) Maoxian station (51MXT) and (b) Pixian station (51PXZ), redrawn from Tao et al. (2013).

ing the shock. The epicenter $\left(31.0^{\circ} \mathrm{N}, 103.4^{\circ} \mathrm{E}\right)$ is denoted by a star in Fig. 1.

Thirty hybrid source models are built (Liu and Tao, 2013); 10 of them are shown in Fig. 8. The motion from each subsource is generated in the same way as mentioned above. The motion from the all sub-sources on rupture plane are superposed by the time histories from every sub-source in the time domain, taking into account the time difference between the rupture in the sub-source and the initial rupture and the time difference between wave arrival times at the ground point from the sub-source and from the rupture start point.

For each station, 30 acceleration time histories are synthesized from these 30 models; the mean values of PGAs are listed in Table 3. The PGAs vary around the mean values with some randomness, and those values are close to the observed PGAs. Response spectra of the 30 motions at each station are shown as gray lines in Fig. 9, in which the black dotted line shows the mean spectrum at each station, and the black solid line shows the spectrum closest to the mean from source model 4 . The acceleration time histories from this source model are chosen as samples for the two stations, shown as the top couple in Fig. 10. The two couples below are $\mathrm{E}-\mathrm{W}$ and $\mathrm{N}-\mathrm{S}$ components of the observed time histories at the stations. The amplitudes are similar, but the durations are different obviously. For Maoxian station, the second and the third sub-shocks are not synthesized.

The response spectra of motions at the two stations from model 4 are shown with those of the observed time histories in Fig. 11, as the black solid line and dotted lines, respectively. The spectra are close to each other at Maoxian station, but they are different between a period of 0.3 and $0.4 \mathrm{~s}$ and longer than $0.7 \mathrm{~s}$ at Pixian station.

\section{Conclusions}

An approach to predict strong ground motion PGA in southwestern China is presented in this paper, by means of a seismology-based attenuation relationship with five regional parameters: $\Delta \sigma, Q_{0}, \eta, R_{1}$, and $R_{2}$. The values of those parameters are estimated from a joint inversion of small earthquake data recorded by regional broadband digital earthquake networks, rather than values from some available references. The objective function of the inversion by $\mu \mathrm{GA}$ is constructed by minimizing the differences between envelopes of Fourier spectra of the small earthquake records and those calculated for the corresponding magnitude and distance of the quakes. The PGA results for $M_{\mathrm{w}} 5.0$ and 6.0 pass through clusters of the limited strong motion data observed in the regions as a whole. The result for Sichuan $M_{\mathrm{w}} 7.0$ is higher than the observed data, since there are no strong motion data in the range of $M_{\mathrm{w}}>6.5$. From this kind of blind test, since in the process of inversion, strong ground motion data are never adopted, the feasibility of this approach is shown for regions without enough strong ground motion data. Furthermore, the regional five parameters are adopted to predict near-fault strong ground motion of a large earthquake $M 8.0$ by motion synthesis, though for the case study at two rock stations in Sichuan, the motion is recorded during the Wenchuan Earthquake. For each station, 30 acceleration time histories are synthesized from 30 finite fault hybrid source models. The results show that PGA can be predicted very well by the mean and that the average spectra are good. The amplitudes of time histories are also similar to the records, but their envelopes are not in accordance with those observed. 
Table 3. Synthesized PGA and observed PGA at two rock stations (gal).

\begin{tabular}{|c|c|c|}
\hline Model & $\begin{array}{l}\text { Maoxian } \\
\text { station }\end{array}$ & $\begin{array}{l}\text { Pixian } \\
\text { station }\end{array}$ \\
\hline 1 & 411.0 & 120.7 \\
\hline 2 & 290.5 & 156.3 \\
\hline 3 & 285.0 & 123.3 \\
\hline 4 & 340.9 & 109.9 \\
\hline 5 & 265.4 & 140.8 \\
\hline 6 & 284.8 & 152.5 \\
\hline 7 & 330.9 & 141.5 \\
\hline 8 & 295.2 & 137.3 \\
\hline 9 & 312.6 & 153.4 \\
\hline 10 & 234.3 & 142.3 \\
\hline 11 & 338.3 & 123.9 \\
\hline 12 & 244.6 & 149.8 \\
\hline 13 & 305.6 & 133.6 \\
\hline 14 & 315.5 & 143.9 \\
\hline 15 & 349.7 & 102.6 \\
\hline 16 & 310.8 & 135.2 \\
\hline 17 & 369.0 & 160.4 \\
\hline 18 & 337.6 & 128.7 \\
\hline 19 & 285.8 & 122.1 \\
\hline 20 & 314.4 & 131.8 \\
\hline 21 & 418.3 & 124.0 \\
\hline 22 & 247.8 & 124.0 \\
\hline 23 & 357.8 & 109.9 \\
\hline 24 & 262.0 & 145.8 \\
\hline 25 & 343.1 & 113.2 \\
\hline 26 & 284.3 & 108.5 \\
\hline 27 & 301.8 & 128.5 \\
\hline 28 & 284.3 & 181.4 \\
\hline 29 & 336.1 & 123.2 \\
\hline 30 & 222.6 & 138.6 \\
\hline Mean value of 30 models & 309 & 134 \\
\hline Observed PGA in an E-W direction & 307 & 121 \\
\hline Observed PGA in an $\mathrm{N}-\mathrm{S}$ direction & 302 & 142 \\
\hline
\end{tabular}

Acknowledgements. This work was financially supported by National Nature Science Foundation of China 51178435, 51478443 and 51178151 and International Science \& Technology Cooperation Program of China 2011DFA21460.

Edited by: F. Masci

Reviewed by: G. De Luca and one anonymous referee

\section{References}

Atkinson, G. M.: Attenuation of strong ground motion in Canada from a random vibrations approach, Bull. Seismol. Soc. Am., 74, 2629-2653, 1984.

Atkinson, G. M.: Earthquake source spectra in the Eastern North America, Bull. Seismol. Soc. Am., 83, 1778-1798, 1993.
Atkinson, G. M. and Boore, D. M.: Ground-motion relation for Eastern North America, Bull. Seismol. Soc. Am., 85, 17-30, 1995.

Atkinson, G. M. and Mereu, R. F.: The shape of ground motion attenuation curves in Southeastern Canada, Bull. Seismol. Soc. Am., 82, 2014-2031, 1992.

Beresnev, I. A. and Atkinson, G.: Stochastic finite-fault modeling of ground motions from the 1994 Northridge, California, earthquake, I. Validation on rock sites, Bull. Seismol. Soc. Am., 88, 1392-1401, 1998.

Boore, D. M.: Stochastic simulation of high-frequency ground motions based on seismological models of the radiated spectra, Bull. Seismol. Soc. Am., 73, 1865-1894, 1983.

Boore, D. M.: Simulation of ground motion using the stochastic method, Pure Appl. Geophys., 160, 635-676, 2003.

Boore, D. M. and Atkinson, G. M.: Stochastic prediction of ground motion and spectral response parameters at hard-rock sites in Eastern North America, Bull. Seismol. Soc. Am., 77, 440-467, 1987.

Boore, D. M. and Joyner, W. B.: Site amplifications for generic rock sites, Bull. Seismol. Soc. Am., 87, 327-341, 1997.

Boore, D. M., Stewart, J. P., Seyhan, E., and Atkinson, G. M.: NGAWest 2 equations for predicting PGA, PGV, and $5 \%$ damped PSA for shallow crustal earthquakes, Earthq. Spectra, 30, 1057-1085, 2014.

Bozorgnia, Y., Abrahamson, N. A., Campbell, K. W., Rowshande, B., and Shantz, T: NGA-West2: A comprehensive research program to update ground motion prediction equations for shallow crustal earthquakes in active tectonic regions, Proc. of 15th World Conference on Earthquake Engineering, Lisbon, Portugal, 2012.

Brune, J. N.: Tectonic stress and the spectra of seismic shear waves from earthquakes, J. Geophys. Res., 75, 4997-5009, 1970.

Carroll, D. L.: http://cuaerospace.com/carroll/ga.html, last access: 20 November 2009.

China Earthquake Networks Center, http://www.csndmc.ac.cn/ newweb/index.jsp, last access: June 2011.

China Strong Motion Networks Center, http://www.csmnc.net/, last access: June 2012.

Hanks, T. C. and McGuire, R. K.: The character of high frequency strong ground motion, Bull. Seismol. Soc. Am., 71, 2071-2095, 1981.

Hu, J. F., Cong, L. L., Su, Y. J., and Kang, G. F.: Distribution characteristics of $Q$ value of the $L g$ coda in Yunnan and its adjacent regions, Chinese J. Geophys., 46, 809-813, 2003.

Kanno, T., Narita, A., Morikawa, N., Fujiwara, H., and Fukushima, Y.: A new attenuation relation for strong ground motion in Japan based on recorded data, Bull. Seismol. Soc. Am., 96, 879-897, 2006.

Liu, C.: Attenuation of strong ground motion from random vibration method. Master Dissertation, Harbin Institute of Technology, Harbin, China, 38-55, 2005.

Liu, H. and Tao, X.: Source model of the great Wenchuan Earthquake 8.0 for synthesis of strong ground motion, China Civ. Eng. J., 46, 139-145, 2013.

Mao, Y., Xu, Y., Wang, B., Hu, J. F., and Cong, L. L.: Distribution characteristics of the value $Q$ of $\mathrm{Lg}$ coda in Sichuan and its adjacent regions, J. Seismol. Res., 28, 38-42, 2005. 
McGuire, R. K. and Hanks, T. C.: RMS accelerations and spectral amplitudes of strong ground motion during the San Fernando, California earthquake, Bull. Seism. Soc. Am., 70, 1907-1919, 1980.

Power, M., Chiou, B., Abrahamson, N., Power, M., Chiou, B., Abrahamson, N., Bozorgnia, Y., Shantz, T., and Roblee, C.: An overview of the NGA project, Earthq. Spectra, 24, 3-21, 2008.

Qiao, H. Z., Zhang, Y. J., Zheng, W. Z., and Liu, J.: The inversion of the inelastic coefficient of the medium in North-West of Sichuan province, Seismol. Geomagn. Obs. Res., 27, 1-7, 2006.

Qin, J. Z. and Kan, R. J.: $Q$ values and seismic moments estimates using the coda waves of near earthquakes in the Kunming and surrounding regions, Acta Geophys. Sin., 29, 145-156, 1986.

Si, H. J. and Midorikawa, S.: New attenuation relations for peak ground acceleration and velocity considering effects of fault type and site condition, Proc. of 12th World Conference on Earthquake Engineering, No. 0532, Auckland, New Zealand, 2000.

Su, Y. J., Liu, J., Zheng, S. H., Liu, L. F., Fu, H., and Xu, Y.: $Q$ value of anelastic S-wave attenuation in Yunnan region, Acta Seismol. Sin., 20, 206-212, 2006.
Tao, X. X., Sun, X. D., and Wang, G. X.: A dynamic corner frequency based source spectral model, Proc. of 14th World Conference on Earthquake Engineering, Beijing, China, S02004, 2008.

Tao X. X., Liu H. M., Wang L. Y., and Jiang W.: Synthesis of strong ground motions at two rock stations during the great Wenchuan earthquake from a hybrid source model, Appl. Mech. Mat., 353356, 1923-1929, 2013.

Wu, J. P., Ming, Y. H., and Wang, C. Y.: The $S$ wave velocity structure beneath digital seismic stations of Yunnan province inferred from teleseismic receiver function modeling, Chin. J. Geophys., 44, 228-237, 2001.

Xie, J., Ni, S. D., and Zeng, X. F.: 1D shear wave velocity structure of the shallow upper crust in central Sichuan Basin, Earthq. Res. Sichuan, 2, 20-24, 2012.

Zhang, Y. J., Qiao, H. Z., Cheng, W. Z., and Liu, J.: Attenuation characteristics of the media in Sichuan basin region, J. Seismol. Res., 30, 43-48, 2007. 